\title{
Práticas integrativas e complementares em saúde: interesse da comunidade acadêmica e os desafios do ensino médico
}

\section{Integrative and complementary practices: interest of the academic community and challenges of medical education}

\author{
Luiza Gomes Dantas Gurgel' (10) | luiza-gurgel@hotmail.com \\ Alana Rebeca Bezerra Jessé1 (1) alana.rjesse@gmail.com \\ Djerlly Marques Araújo da Silva (iD) djerlly.marques@gmail.com \\ Pedro Sá Leitão Laporte Alencar ${ }^{1}$ (D) pedrolaporte2@gmail.com \\ Arturo de Pádua Walfrido Jordán' (1) arturojordan@fps.edu.br \\ Nicolas Augusto Alves Daniel² (1) coordenacaopics.recife@gmail.com
}

\section{RESUMO}

Introdução: As Práticas Integrativas e Complementares em Saúde (PICS) abrangem recursos terapêuticos de sistemas médicos complexos de diversas racionalidades médicas. Esse campo de práticas vem ganhando maior visibilidade nos últimos tempos com o aumento da procura por cuidados em saúde que priorizem a abordagem integral do ser humano, estimulando os próprios profissionais de saúde a buscar uma melhor formação.

Objetivo: Este estudo teve como objetivo avaliar o interesse, o grau de conhecimento e a atitude de discentes e docentes do curso de Medicina pelas PICS e os desafios para seu ensino efetivo.

Método: Trata-se de um estudo transversal, descritivo e com abordagem quantitativa, realizado com 214 indivíduos, sendo 21 docentes e 193 discentes do primeiro ao décimo segundo período do curso de Medicina de uma instituição de ensino de saúde de Recife, em Pernambuco. Utilizou-se um questionário em formato on-line que avaliou os sujeitos pesquisados quanto às fontes de aprendizado e ao nível de interesse sobre o tema. Adotou-se também a versão em português do instrumento Integrative Medicine Attitude Questionnaire (IMAQ). Os dados foram submetidos à análise estatística descritiva (frequência e porcentagem), com o auxílio dos softwares R versão 3.4.3. e LibreOffice.

Resultado: Dentre os participantes do estudo, 57,14\% dos docentes e 35,42\% dos discentes afirmaram conhecer a Política Nacional de Práticas Integrativas e Complementares (PNPIC), e $85,71 \%$ dos docentes e $91,7 \%$ dos discentes consideraram as PICS fundamentais para o SUS. A maioria considerou o ensino das PICS importante para a graduação (90,48\% dos docentes e $89,58 \%$ dos discentes). As práticas mais conhecidas foram a ioga, a fitoterapia e a MTC/acupuntura, e as que despertaram maior interesse para aprendizado foram a ioga e a MTC/acupuntura.

Conclusão: Um número elevado de discentes e docentes nunca teve contato com as PICS. No entanto, a maioria tem disposição em recomendar para pacientes e familiares, bem como vontade em aprender sobre o tema. Dessa forma, recomenda-se que mais trabalhos sejam realizados sobre a temática e que isso possa corroborar a sua inclusão na base curricular dos cursos de saúde.

Palavras-chave: Terapias Complementares; Educação Médica; Estudantes de Medicina; Docentes de Medicina; Sistema Único de Saúde.

\section{ABSTRACT}

Introduction: Traditional Complementary and Alternative Medicine (TCAM) encompass therapeutic resources of complex medical systems from different medical rationalities. This field of practice has gained greater visibility in recent times, with the increase in demand for health care that prioritizes a more comprehensive approach of human beings, encouraging the health professionals themselves to seek better training.

Objective: To evaluate the interest, the degree of knowledge and the attitude of students and teachers of the medical course towards the TCAM and the challenges for their effective teaching.

Method: This is a cross-sectional, descriptive study with a quantitative approach, which was carried out with 214 individuals, 21 of which were teachers and 193 students attending the first to the twelfth semesters of the medical course at a health education institution in Recife-PE. An online survey was used to assess the study participants regarding their learning sources and the level of interest on this topic, as well as the Portuguese version of the Integrative Medicine Attitude Questionnaire (IMAQ). The data were submitted to descriptive statistical analysis (frequency and percentage), using software $R$, version 3.4.3. and LibreOffice.

Result: Among the study participants, $57.14 \%$ of teachers and $35.42 \%$ of students were aware of the Nacional TCAM Policy (PNPIC, Politica Nacional de Práticas Integrativas e Complementares), $85.71 \%$ of the teachers and $91.7 \%$ of students considered TCAM to be crucial for Brazilian Unified Health System (SUS). Most considered the teaching of TCAM an important subject for the undergraduate medical course $(90.48 \%$ of teachers and $89.58 \%$ of students). The best known practices were yoga, phytotherapy and TCM/acupuncture and there was greater interest to learn yoga and TCM/acupuncture.

Conclusion: A large number of students and teachers have never had contact with TCAM. However, most are willing to recommend them to patients and family members and are willing to learn about the topic. Thus, it is recommended that more studies be performed on this topic and that it can corroborate for their inclusion in health courses' curriculum.

Keywords: Complementary Therapies; Education, Medical; Students, Medical; Medical Faculty; Unified Health System.

${ }^{1}$ Faculdade Pernambucana de Saúde, Recife, Pernambuco, Brasil.

${ }^{2}$ Prefeitura Municipal do Recife, Recife, Pernambuco, Brasil.

Editora-chefe: Rosiane Viana Zuza Diniz. | Editora associada: Ana Cláudia Santos Chazan.

Recebido em 08/06/21; Aceito em 25/10/21. | Avaliado pelo processo de double blind review. 


\section{INTRODUÇÃO}

As Práticas Integrativas e Complementares em Saúde (PICS) são um conjunto heterogêneo de práticas, saberes e recursos terapêuticos que englobam sistemas médicos de diversas racionalidades. Esse campo é denominado pela Organização Mundial da Saúde (OMS) de Medicina Tradicional e Complementar/Alternativa (MT/MCA) e envolve abordagens que buscam estimular mecanismos naturais de recuperação da saúde e prevenção de agravos por técnicas que enfatizam o acolhimento e a visão integral do homem em seus âmbitos físico, mental, social e espiritual'.

No final da década de 1970, a OMS criou o Programa de Medicina Tradicional para formular políticas na área². Desde então, há o compromisso de incentivar os Estados-membros a implementar políticas públicas para o uso racional da MT/MCA nos sistemas de atenção à saúde, bem como o desenvolvimento de estudos para melhor conhecimento de sua segurança e qualidade ${ }^{1}$. Foi nessa perspectiva que, no Brasil, em 2006, foi criada a Política Nacional de Práticas Integrativas e Complementares (PNPIC) no Sistema Único de Saúde (SUS). A PNPIC, dentro de 29 práticas regulamentadas atualmente, legitimou a oferta pública de atividades que já eram utilizadas pela população³.

A PNPIC no SUS ainda tem apoio insuficiente, o que significa baixo incentivo financeiro, poucos investimentos na capacitação dos profissionais e avaliação e monitoramento precários. Entretanto, já é de reconhecimento da OMS que as PICS apresentam eficácia em áreas como saúde mental, prevenção e tratamento de doenças não transmissíveis e melhora da qualidade de vida de pessoas com doenças crônicas, assim como da população em envelhecimento. Mesmo com todos os desafios, a procura pelas técnicas vem aumentando. A busca pelo equilíbrio da saúde por meio de intervenções com enfoque numa visão holística vem crescendo cada dia mais, principalmente quando se almeja construir um sistema de saúde que visa, em um dos seus três princípios básicos, à integralidade do cuidado ${ }^{4}$.

É fundamental destacar a realidade das PICS em Recife, no estado de Pernambuco, local da instituição avaliada neste estudo. O histórico de atuação na área antecede a própria PNPIC, sendo pioneira no fornecimento dessas práticas vinculadas à Estratégia Saúde da Família (ESF), como na Unidade de Cuidados Integrais à Saúde (UCIS) Professor Guilherme Abath, fundada em 2002 e que hoje é campo de prática da residência médica em saúde da família, como também há a Política Municipal de Práticas Integrativas e Complementares em Saúde de Recife, criada em 2012, que fortalece ainda mais a presença dessas práticas na rede de saúde local ${ }^{5,6}$.

Pensando na carência de pesquisas e no crescente interesse da população pelas práticas, é importante que esse tema seja levado aos ambientes acadêmicos. No contexto prático, é vista, contudo, uma realidade que vai de encontro à crescente demanda da população por terapias não convencionais: veem-se muitos médicos que não estão aptos para responder às questões trazidas pelos pacientes sobre o uso de PICS, nem sobre como são os mecanismos de ação, as indicações, as interações medicamentosas ou mesmo os efeitos adversos dessas terapias ${ }^{7}$.

Para a compreensão dos desafios do ensino das PICS, destaca-se que preconceitos e pouco conhecimento estão relacionados com o ensino médico adotado no Brasil. Apesar de mudanças com as Diretrizes Curriculares Nacionais (DCN), ainda segue com influências da reforma flexneriana, na América do Norte, no início do século $\mathrm{XX}$, que valorizava uma formação médica vinculada à clínica laboratorial, ao consumo de exames e procedimentos e à fragmentação do saber em busca de uma formação mais eficiente. Devido à mercantilização da saúde, esse modelo se disseminou pelo Ocidente, e, então, as PICS foram excluídas da graduação por serem consideradas "não científicas"8.

Apesar de no Brasil haver insuficientes pesquisas acerca do ensino das PICS na graduação, mundialmente já há exemplos de escolas médicas que as abarcam, inclusive com o desenvolvimento de instrumentos de avaliação das atitudes diante desse tema, como o Integrative Medicine Attitude Questionnaire (IMAQ) ${ }^{9}$.

Mesmo com um currículo médico que não supre a necessidade de ensino sobre o tema, constatou-se que, de maneira geral, os estudantes de Medicina apresentam atitudes favoráveis em relação às PICS e têm o desejo de ver conteúdos dessa temática. Além disso, também consideram importante a oferta das PICS nos serviços de saúde e muitos acreditam na possibilidade de integração à medicina convencional ${ }^{10}$.

Nesse sentido, este estudo procurou avaliar o interesse, o grau de conhecimento e a atitude de discentes e docentes do curso de Medicina em relação às PICS e os desafios para seu ensino efetivo. Esses conhecimentos possibilitarão identificar questões importantes para a modificação do cenário de carência teórico-prática sobre as PICS da educação médica.

\section{MÉTODO}

Trata-se de um estudo transversal, descritivo e com abordagem quantitativa, realizado em uma Faculdade de Medicina de Recife que desenvolve seu projeto pedagógico ancorada nos princípios da metodologia ativa, por meio do método de Aprendizagem Baseada em Problemas (ABP), em compromisso com as diretrizes preconizadas pelo SUS. O referido curso é programado para o aprendizado em ambiente de laboratórios do primeiro ao quarto período, em ambiente ambulatorial hospitalar do quinto ao oitavo período e em cenário hospitalar, com estágio supervisionado, do nono ao $12^{\circ}$ período. 
A população de estudo foi composta pelo corpo discente e docente do curso de Medicina referido do primeiro ao $12^{\circ}$ semestre letivo, sendo 1.084 o total de estudantes regularmente matriculados na instituição, em qualquer etapa letiva do curso, e 88 o total de docentes vinculados a atividades acadêmicas dentro dos referidos períodos letivos analisados (dados de 2019). A amostra foi definida por conveniência, do tipo não probabilística, e constituída por 214 indivíduos, sendo 21 docentes e 193 discentes.

Incluíram-se no estudo pessoas com idade acima de 18 anos, estudantes devidamente matriculados no referido curso de Medicina no período de realização da pesquisa e que estivessem cursando quaisquer das etapas do primeiro ao $12^{\circ}$ semestre letivo, e docentes do referido curso, dentro do período vigente do presente estudo. Excluíram-se da análise os estudantes em situação de trancamento de matrícula.

Para a coleta de dados, utilizou-se um questionário composto por: 1. dados sociodemográficos e ocupacionais; 2. conhecimento geral acerca das PICS no SUS e as opiniões e experiências vivenciadas tanto em ambiente acadêmico como na prática médica, com todas as PICS contidas na PNPIC; e 3. o IMAQ?.

O IMAQ é um instrumento que surgiu da necessidade de haver uma maneira autoaplicável, confiável e psicometricamente capaz de medir a atitude de estudantes perante a medicina integrativa para fornecer aos educadores uma ferramenta útil para implementar, avaliar e revisar de forma sensata os currículos de medicina complementar. Em 2015, o questionário foi validado para o português ${ }^{9}$, versão utilizada no presente estudo. Utilizase o formato da escala Likert modificada, contendo 7 pontos variando de $-3 a+3$, sendo -3 equivalente a discordo totalmente e +3 concordo totalmente. Esse instrumento apresenta uma estrutura dividida em dois fatores: o primeiro refere-se à abertura para novas ideias e paradigmas, contendo 21 itens - o qual é denominado Openness -, e o segundo está relacionado a valores de introspecção do profissional de saúde e à relação entre médico e paciente - fator chamado Relationship - contendo oito itens. Portanto, o IMAQ contém, no total, 29 itens e pontua de forma negativa ou positiva.

$\mathrm{Na}$ construção do questionário on-line, utilizou-se o LimeSurvey, e enviaram-se e-mails para toda a comunidade acadêmica do curso. Outra estratégia utilizada para ampliar o número de respondentes foi o contato por um aplicativo de mensagem.

A formação da base de dados e sua análise foram efetuadas com uso do programa LibreOffice. Organizaram-se os dados em três momentos de análise: 1. descrição do perfil dos respondentes: considerando idade, gênero do participante, atividade realizada na Instituição, período de curso do discente e formação médica do docente; 2 . conhecimento e interesse de docentes e discentes: conhecimento das PICS que compõem a PNPIC, identificação das práticas conhecidas, das abordadas na graduação, das que há interesse em aprender e das que o participante já possui formação complementar prévia, local de obtenção de conhecimento sobre PICS, nível de satisfação com os conhecimentos fornecidos no curso, nível de elucidação e alcance de objetivos de ensino e aprendizado na área, consideração de PICS como fundamentais para SUS, crença em torno da necessidade de ensino das PICS na graduação médica, identificação dos participantes que as recomendariam para pacientes e/ou familiares, identificação de docentes com interesse em abordar o tema em atividades curriculares e com interesse por capacitação para o ensino das PICS; e 3. atitudes perante as PICS, como abertura ao tema e relação médico-paciente.

Para a análise estatística, as variáveis categóricas foram calculadas como frequências absolutas (contagens) e relativas (porcentagens). Os dados provenientes do questionário eletrônico foram submetidos à análise estatística descritiva (frequência e porcentagem), com o auxílio dos softwares $\mathrm{R}$ versão 3.4.3. e LibreOffice. Os resultados são apresentados em forma de tabelas. Quanto aos resultados obtidos pelo IMAQ, é possível observar se há atitudes positivas acerca de PICS quanto maior for a pontuação.

A presente pesquisa atende às resoluções nos $466 / 2012$ e 510/2016 do Conselho Nacional de Saúde e foi aprovada por Comitê de Ética em Pesquisa da FPS sob o parecer n 3.495.554. Todos os participantes que preencheram os critérios de elegibilidade consentiram em fazer parte do estudo mediante concordância do Termo de Consentimento Livre e Esclarecido (TCLE), incluído no questionário on-line.

\section{RESULTADOS}

A amostra foi composta por 193 discentes e 21 docentes. Esse número de estudantes corresponde a 17,8\% dos 1084 discentes matriculados no curso de Medicina do primeiro ao décimo segundo semestre letivo, durante o período vigente da presente pesquisa. A quantidade de docentes que responderam ao questionário corresponde a $23,8 \%$ do corpo de 88 tutores do curso de Medicina da faculdade. A distribuição de gênero foi de 16 docentes $(76,2 \%)$ e 134 discentes $(69,4 \%)$ do sexo feminino e cinco docentes $(23,8 \%)$ e 59 discentes $(30,6 \%)$ do sexo masculino. Quanto à fase do curso, 87 discentes $(45,1 \%)$ cursavam do nono e o $12^{\circ}$ semestre; $58(30,1 \%)$, do primeiro ao quarto semestre; e 48 (24,9\%), do quinto e o oitavo semestre. Referente às especialidades na área de saúde, cinco dos tutores que compõem a amostra trabalham na atenção primária à saúde $(23,8 \%)$, cinco são pediatras $(23,8 \%)$, quatro (19\%) atuam na área de ginecologia e obstetrícia, e três $(14,3 \%)$ trabalham em geriatria ou em alguma especialidade cirúrgica. Dentro das 
atividades exercidas na instituição, 12 (57,1\%) são tutores dos módulos regulares, quatro (19\%) são tutores dos módulos da Prática de Atenção Primária e cinco (23,9\%) exercem alguma atividade de coordenação dentro do curso.

No tocante ao conhecimento acerca das PICS, 12 (57,1\%) docentes e 69 (35,8\%) discentes afirmaram conhecer a PNPIC. A ioga foi a prática mais conhecida tanto pelos docentes $(\mathrm{N}=$ $17 ; 80,9 \%$ ) quanto pelos discentes ( $N=159 ; 82,4 \%$ ), seguida de plantas medicinais e fitoterapia para os docentes $(N=16 ; 76,2 \%)$ e meditação para os discentes ( $N=154 ; 79,8 \%)$. A fonte de conhecimento sobre as PICS referida por sete docentes $(33,3 \%)$ foi por meio de profissionais não vinculados à faculdade, quatro $(19,1 \%)$ obtiveram por meio de estudos individuais, três (14,3\%) adquiriram nos meios de comunicação de massa, três (14,3\%) nunca tiveram contato e dois $(9,6 \%)$ obtiveram contato com esse conteúdo em vivências na faculdade. Quanto aos estudantes, $110(57,4 \%)$ obtiveram conhecimento sobre as PICS a partir de atividades acadêmicas vinculadas à faculdade, enquanto 38 $(19,7 \%)$ afirmaram nunca ter tido contato com o assunto.

Dos 110 discentes que mencionaram ter obtido conhecimento sobre as PICS dentro de alguma vivência acadêmica desenvolvida pela faculdade, 52 (47,3\%) não concordaram ou discordaram fortemente de que os conhecimentos fornecidos pela instituição foram satisfatórios no tocante à segurança na aplicação prática. Além disso, 52 discentes (47,3\%) afirmaram que os conhecimentos fornecidos pela faculdade não tiveram objetivos de ensino e aprendizagem suficientemente elucidativos.

Entre as práticas mais abordadas durante o período da graduação dos docentes entrevistados, destacaram-se a arteterapia ( $\mathrm{N}=6 ; 28,57 \%)$, a homeopatia ( $\mathrm{N}=5 ; 23,8 \%)$, a medicina tradicional chinesa (MTC)/acupuntura ( $N=5 ; 23,8 \%$ ), a fitoterapia ( $N=4 ; 19 \%)$ e a aromaterapia ( $N=4 ; 19 \%)$. Os discentes entrevistados apontaram a abordagem em meio acadêmico da homeopatia ( $N=53 ; 27,5 \%)$, da MTC/acupuntura ( $N=46 ; 23,8 \%$ ), da fitoterapia ( $N=43 ; 22,3 \%)$, da meditação $(\mathrm{N}=37 ; 19,1 \%)$ e da arteterapia ( $\mathrm{N}=35 ; 18,1 \%)$.

Entre as PICS mais utilizadas tanto pelos docentes quanto pelos discentes entrevistados, destacaram-se: meditação 101 (52,3\%) discentes e 9 (42,8\%) docentes; ioga - 76 (39,4\%) discentes e $8(38,1 \%)$ docentes; fitoterapia - $67(34,7 \%)$ discentes e 7 (33,3\%) docentes; MTC/acupuntura - 51 (26,4\%) discentes e $8(38,1 \%)$ docentes; e homeopatia - 45 (23,3\%) discentes e 7 $(33,3 \%)$ docentes.

Quanto ao interesse em receber capacitação teórica e prática para o ensino das PICS na atividade de docência, 19 $(90,5 \%)$ dos docentes entrevistados sinalizaram o interesse tanto em aprender quanto em abordar sobre as PICS com os estudantes nas atividades curriculares. Dos docentes entrevistados, três
(14,3\%) já tinham formação complementar em meditação, três $(14,3 \%)$ em imposição de mãos e dois $(9,6 \%)$ em reiki. Dos discentes, seis $(3,1 \%)$ tinham formação complementar em arteterapia, cinco $(2,6 \%)$ em reiki e três $(1,5 \%)$ em meditação.

Entre as práticas que despertaram maior interesse de aprendizado para os docentes, destacaram-se musicoterapia $(\mathrm{N}=7 ; 33,33 \%)$, ioga ( $\mathrm{N}=6 ; 28,6 \%)$, MTC/acupuntura $(\mathrm{N}=6$; $28,6 \%)$, homeopatia ( $N=5 ; 23,8 \%$ ) e meditação ( $N=5 ; 23,81 \%)$. Já para os discentes, as práticas que mais incitaram a vontade de aprender foram MTC/acupuntura ( $\mathrm{N}=92 ; 47,6 \%)$, ioga ( $\mathrm{N}=78$; $40,4 \%)$, fitoterapia ( $N=76 ; 39,4 \%)$, meditação $(N=76 ; 39,4 \%)$ e quiropraxia ( $\mathrm{N}=74 ; 39,4 \%)$.

A maioria dos entrevistados considerou que as PICS são fundamentais para o SUS: $177(91,7 \%)$ dos discentes e $18(85,7 \%)$ dos docentes. Além disso, 173 (89,6\%) discentes e 19 (90,5\%) docentes consideraram que o ensino das PICS é necessário na graduação médica. Todos os docentes e discentes entrevistados afirmaram que recomendariam alguma das PICS para seus pacientes e/ou familiares.

Dos resultados obtidos por meio do IMAQ, obteve-se uma pontuação média de 31,17 (sendo o valor mínimo-29 e o máximo 75), com mediana de 33,5 e desvio padrão (DP) de 17,56. Quanto à categorização nos dois fatores, o item Openness, referente à abertura para novas ideias e paradigmas, apresentou média de 17,81 (valor mínimo-25 e máximo 53), com mediana de 18 e DP de 13,29 , enquanto no item Relationships, que trata da introspecção e relação médico-paciente, a média foi de 13,36 (valor mínimo-15 e máximo 24), com mediana de 14 e DP de 6,92.

Tabela 1. Conhecimento e interesses dos docentes do curso de Medicina acerca das PICS. Recife, Pernambuco, Brasil, em 2020.

\begin{tabular}{lcc}
\hline \multicolumn{1}{c}{ Característica } & N & $\%$ \\
\hline Total de docentes da amostra & 21 & 100 \\
\hline Área de atuação/especialização profissional & & \\
Pediatria & 5 & 23,8 \\
Atenção primária à saúde & 5 & 23,8 \\
Ginecologia e obstetrícia & 4 & 19,0 \\
Geriatria & 3 & 14,3 \\
Cirurgia & 3 & 14,3 \\
\hline Conhece a PNPIC & 12 & 57,1 \\
\hline PICS mais conhecidas & & \\
loga & 17 & 80,9 \\
Plantas medicinais e fitoterapia & 16 & 76,2 \\
Meditação & 15 & 71,4 \\
Homeopatia & 15 & 71,4 \\
Musicoterapia & 15 & 71,4 \\
\hline
\end{tabular}


Tabela 1. (Continuação) Conhecimento e interesses dos docentes do curso de Medicina acerca das PICS. Recife, Pernambuco, Brasil, em 2020.

\begin{tabular}{llc}
\hline \multicolumn{1}{c}{ Característica } & N & \% \\
\hline $\begin{array}{l}\text { Fonte de conhecimento sobre as PICS } \\
\text { Profissionais não vinculados à instituição }\end{array}$ & 7 & 33,3 \\
$\begin{array}{l}\text { de ensino } \\
\text { Estudo individuais }\end{array}$ & 4 & 19,0 \\
Meios de comunicação de massa & 3 & 14,3 \\
Nunca teve contato & 3 & 14,3 \\
Congressos, simpósios, conferências ou & 2 & 9,5 \\
outros eventos científicos & & \\
Módulo teórico em atenção primária & 1 & 4,8 \\
Exposições & 1 & 4,8 \\
\hline
\end{tabular}

\section{PICS abordadas durante a graduação}

\begin{tabular}{lll} 
Arteterapia & 6 & 28,6 \\
Homeopatia & 5 & 23,8 \\
MTC/acupuntura & 5 & 23,8 \\
Plantas medicinais e fitoterapia & 4 & 19,0 \\
Aromaterapia & 4 & 19,0 \\
\hline PICS mais utilizadas & & \\
Meditação & 9 & 42,8 \\
MTC/acupuntura & 8 & 38,1 \\
loga & 8 & 38,1 \\
Plantas medicinais e fitoterapia & 7 & 33,3 \\
Homeopatia & 7 & 33,3 \\
Reiki & 7 & 33,3 \\
\hline Interesse em abordar sobre as PICS nas & 19 & 90,5 \\
atividades de docência & 19 & 90,5 \\
\hline Interesse em receber capacitação para o \\
ensino das PICS nas atividades de docência
\end{tabular}

\begin{tabular}{lll}
\hline Possui formação complementar & & \\
Meditação & 3 & 14,3 \\
Imposição de mãos & 3 & 14,3 \\
Reiki & 2 & 9,6 \\
Plantas medicinais e fitoterapia & 1 & 4,7 \\
Shantala & 1 & 4,7 \\
\hline
\end{tabular}

\section{Gostaria de aprender}

\begin{tabular}{llc} 
Musicoterapia & 7 & 33,3 \\
loga & 6 & 28,6 \\
MTC/acupuntura & 6 & 28,6 \\
Meditação & 5 & 23,8 \\
Homeopatia & 5 & 23,8 \\
\hline $\begin{array}{l}\text { Considera que as PICS são fundamentais para } \\
\text { o SUS }\end{array}$ & 18 & 85,7 \\
\hline $\begin{array}{lll}\text { Acredita que o ensino das PICS é necessário na } \\
\text { graduação médica }\end{array}$ & \multicolumn{2}{|c}{90,5} \\
\hline $\begin{array}{l}\text { Recomendaria para pacientes e/ou familiares } \\
\text { 21 }\end{array}$ & 100 \\
\hline
\end{tabular}

Tabela 2. Conhecimento e interesses dos discentes do curso de Medicina acerca das PICS. Recife, Pernambuco, Brasil, em 2020.

\begin{tabular}{lcc}
\hline \multicolumn{1}{c}{ Característica } & N & $\%$ \\
\hline Total de discentes da amostra & 193 & 100 \\
\hline Conhece a PNPIC & 69 & 35,8 \\
\hline PICS mais conhecidas & & \\
loga & 159 & 82,4 \\
Meditação & 154 & 79,8 \\
MTC/acupuntura & 145 & 75,1 \\
Plantas medicinais e fitoterapia & 130 & 67,3 \\
Musicoterapia & 124 & 64,2 \\
\hline
\end{tabular}

Fonte de conhecimento sobre as PICS

Exposições $\quad 57 \quad 29,5$

$\begin{array}{lll}\text { Nunca teve contato } & 38 & 19,7\end{array}$

Módulo teórico em atenção primária $\quad 33 \quad 17,1$

Meios de comunicação de massa $\quad 15 \quad 7,8$

$\begin{array}{lll}\text { Módulo de tutoria } & 11 & 6,7\end{array}$

$\begin{array}{lll}\text { Eventos científicos } & 11 & 5,7\end{array}$

$\begin{array}{lll}\text { Atividade de integração entre turmas } & 6 & 3,1\end{array}$

$\begin{array}{lll}\text { Outros } & 6 & 3,1\end{array}$

Profissionais não vinculados à instituição de $\quad \begin{array}{lll}2,6 & 6\end{array}$ ensino

21,0

Rodízio em Unidade de Saúde da Família vinculada à instituição de ensino

Os conhecimentos fornecidos pelas vivências na faculdade são satisfatórios para ter segurança na 110 sua aplicação prática

Concordam/concordam fortemente $\quad 27 \quad 24,5$

$\begin{array}{lll}\text { Neutros } & 31 & 28,2\end{array}$

Discordam/discordam fortemente $52 \quad 47,3$

Os objetivos de ensino e aprendizado desse tema 110

foram elucidados e alcançados

Concordam/concordam fortemente $\quad 27 \quad 24,5$

$\begin{array}{lll}\text { Neutros } & 31 & 28,2\end{array}$

Discordam/discordam fortemente $52 \quad 47,3$

PICS abordadas durante a graduação

$\begin{array}{lll}\text { Homeopatia } & 53 & 27,5\end{array}$

MTC/acupuntura $46 \quad 23,8$

Plantas medicinais e fitoterapia $\quad 43 \quad 22,3$

$\begin{array}{lll}\text { Meditação } & 37 & 19,1\end{array}$

$\begin{array}{lll}\text { Arteterapia } & 35 & 18,1\end{array}$

PICS mais utilizadas

$\begin{array}{lll}\text { Meditação } & 101 & 52,3\end{array}$

$\begin{array}{lll}\text { loga } & 76 & 39,4\end{array}$

$\begin{array}{lll}\text { Plantas medicinais e fitoterapia } & 67 & 34,7\end{array}$

$\begin{array}{lll}\text { MTC/acupuntura } & 51 & 26,4\end{array}$

Homeopatia $\quad 45 \quad 23,3$ 
Tabela 2. (Continuação) Conhecimento e interesses dos discentes do curso de Medicina acerca das PICS. Recife, Pernambuco, Brasil, em 2020.

\begin{tabular}{lcc}
\hline \multicolumn{1}{c}{ Característica } & N & $\%$ \\
\hline Gostaria de aprender & 92 & 47,6 \\
MTC/acupuntura & 78 & 40,4 \\
loga & 76 & 39,4 \\
Plantas medicinais e fitoterapia & 76 & 39,4 \\
Meditação & 74 & 39,4 \\
Quiropraxia & & \\
\hline Possui formação complementar & 6 & 3,1 \\
Arteterapia & 5 & 2,6 \\
Reiki & 3 & 1,5 \\
Meditação & 3 & 1,5 \\
Terapia de florais & 2 & 1,0 \\
Plantas medicinais e fitoterapia & 2 & 1,0 \\
MTC/acupuntura & 177 & 91,7 \\
\hline Considera que as PICS são fundamentais parao & & \\
SUS & 173 & 89,6 \\
\hline Acredita que o ensino das PICS é necessário na & 193 & 100 \\
\hline graduação médica & \multicolumn{2}{|c}{} \\
\hline Recomendaria para pacientes e/ou familiares & & \\
\hline
\end{tabular}

\section{DISCUSSÃO}

Quanto ao perfil dos participantes, identificou-se uma maioria do sexo feminino, dado que condiz com o cenário nacional do processo de feminização do trabalho médico ${ }^{11}$. Sobre os docentes, constatou-se um predomínio de atuação na área de atenção primária, enquanto os estudantes se encontravam principalmente no internato do curso, contexto que favorece uma maior familiarização com as PICS, já que são oferecidas sobretudo no nível primário de atenção e esse grupo possui maior vivência nesse ambiente. Nele, os valores são voltados para uma saúde em perspectiva comunitária e integral, e ressoam com as diferentes racionalidades que abarcam as PICS $^{8}$. Assim, afirma-se que são participantes que representam parcela do corpo acadêmico com maior chance de proximidade com PICS na graduação.

Quando se abordou a PNPIC ${ }^{12}$, verificou-se uma lacuna de conhecimento do corpo acadêmico referente à política. Esse cenário chama a atenção quando se considera o município da instituição em questão. Com unidades de saúde como a UCIS e políticas municipais próprias para $\operatorname{PICS}^{5,6,8}$ que antecederam à PNPIC ou que se fortaleceram desde então, observou-se que há uma desvinculação do ensino com os serviços de saúde, além

Figura 1. Atitudes do corpo acadêmico em relação às PICS a partir do IMAQ. Recife, Pernambuco, Brasil, em 2020.

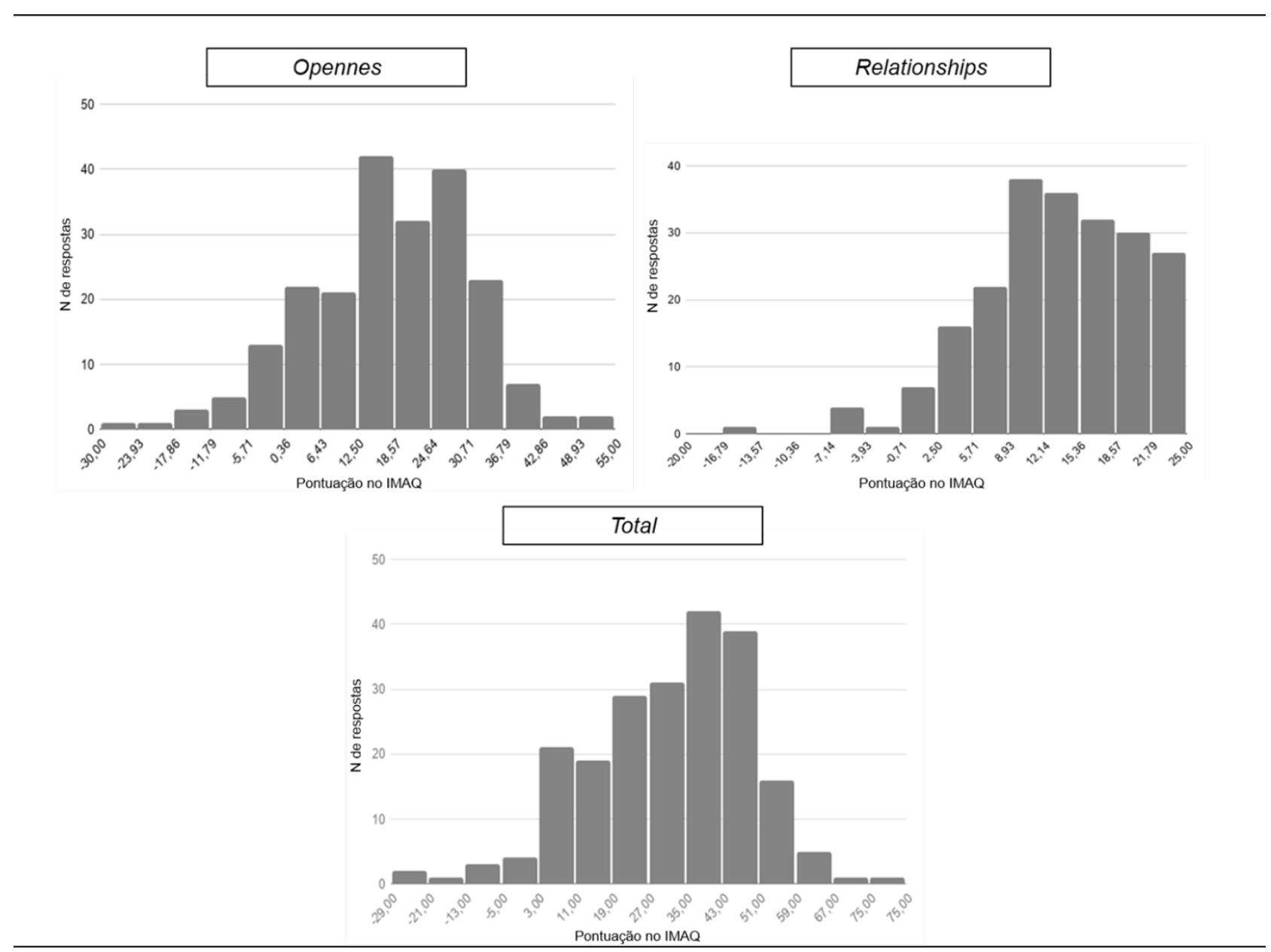


de desconhecimento sobre os cuidados a que a população já tem acesso e que tende a procurar cada vez mais ${ }^{13}$. Para que haja uma rede de saúde que abarque idealmente as PICS, é necessária a formação com esse intuito, vinculando ensinoserviço-comunidade. Apesar disso, os participantes têm atitudes favoráveis para uma inserção do tema na graduação, visto que a maioria considera as PICS como fundamentais e apoia que seu ensino tenha maior inserção na grade curricular, algo também recomendado porpesquisadores ${ }^{13}$.

Outro desafio identificado foi quanto ao ambiente de obtenção de conhecimento sobre o tema pelos docentes: a maioria recorreu a estudos individuais ou contatos externos à instituição, com grande parcela tendo contato superficial pelos meios de comunicação em massa ou mesmo nunca tendo tido contato. Além disso, há a afirmação dos discentes que colocam em xeque a qualidade do ensino fornecido, já que parte deles não sente segurança para a aplicação prática dos conhecimentos obtidos e afirmaram não haver objetivos de ensino e aprendizagem elucidados para guiar os estudos. Soma-se a esse quadro, porém, o fato de todos os participantes afirmarem que recomendariam ao menos alguma das PICS. Portanto, considerando o que ditam as $\mathrm{DCN}^{14}$ para a formação médica adequada, percebem-se uma fragilidade e falta de preparo sobre o tema, com potenciais riscos para a atuação médica, já que não há conhecimentos necessários quanto aos possíveis riscos ou efeitos adversos, para fazer a recomendação correta dessas terapias ${ }^{7,15}$.

Há exemplos de faculdades e programas de residências médicas com inclusão de PICS na grade de ensino ${ }^{16}$, mas o preparo de tutores e preceptores é crucial. Foi observado no estudo que há interesse em capacitação sobre o tema, o que remete a atitudes positivas para a qualificação profissional. Uma pequena parcela dos tutores e preceptores tem formação complementar em alguma prática, mas a identificação da existência desses se torna importante quando se pensa na necessidade de colaboração entre profissionais para haver maior quebra de preconceitos e aplicabilidade das PICS.

Em relação a cada prática individualizada, identificouse um conhecimento geral maior em torno das mais bem difundidas e com maior quantidade de pesquisas clínicas comprovando seus benefícios para a saúde ${ }^{5,6}$, caso da ioga, das plantas medicinais e da meditação, como também de práticas que no Brasil têm programas de residência médica firmados, caso da homeopatia e MTC/acupuntura ${ }^{13}$. Cabe também fazer um paralelo com as práticas mais utilizadas pelos participantes e com o interesse em aprendê-las, que são praticamente as mesmas, mostrando que há um grau de relação entre o uso próprio de certas práticas despertando interesse pelo aprendizado e atitudes favoráveis à sua utilização e recomendação.
Quanto aos dados referentes ao IMAQ, vê-se que a pontuação média total positiva, com desvio padrão que não demonstra variações extremas entre mínimo e máximo, reforça a existência de atitudes favoráveis a abordagens mais holísticas. Considerando-se individualmente os fatores desse instrumento, em relação à abertura para novas ideias e paradigmas, pode-se afirmar que, apesar do valor médio positivo, ainda é pouco expressivo em relação ao valor máximo possível, o que remete à resistência a temas que se afastam da abordagem exclusivamente biomédica. Já em relação ao fator de introspecção e à relação médico-paciente, o IMAQ possibilita dizer que proporcionalmente há atitudes mais positivas em comparação com abertura para temas como PICS.

É importante salientar que os resultados referentes à população estudada não podem ser compreendidos como representativos de todo o universo do público-alvo, devido ao viés de seleção identificado durante a aplicação do questionário. Por se tratar de um estudo do tipo survey aplicado por meio de questionário on-line em plataforma virtual própria fornecida pela instituição de ensino, houve a facilidade tecnológica de disseminação do questionário para todos os discentes e docentes repetidas vezes, mas a formatação virtual restrita fez com que o questionário inicialmente pensado se tornasse mais longo, fator contribuinte para a taxa de não resposta a ele $\mathrm{e}^{17,18}$, como também houve discrepâncias entre quantidade de respondentes dos diferentes períodos do curso. Portanto, os dados aqui analisados são representativos apenas da população estudada, não devendo ser extrapolados nem tomados como realidade concreta da população em geral.

A avaliação dos conhecimentos, dos interesses e das atitudes de docentes e discentes acerca das PICS é um campo pouco explorado pelos profissionais de saúde no processo de educação médica. Os resultados apresentados por esta pesquisa condizem com estudos prévios $^{14}$ que evidenciaram conhecimento pouco abrangente por parte de profissionais e estudantes de saúde em relação às PICS. Isso se relaciona com a perpetuação de preconceitos originados nas reformas da educação médica do século passado e a falta de abordagem do tema ao longo da graduação ${ }^{13,15}$ resultante dos reflexos do Relatório Flexner e de sua influência histórica na educação brasileira. Atualmente em que se busca cada vez mais quebrar com o modelo de saúde centrada na doença e o ensino exclusivamente hospitalar, com a modificação do cenário educacional em que os níveis primário e secundário de saúde passaram ter maior espaço na graduação e a humanização e gestão do cuidado se tornaram pautas centrais, há espaço para o crescimento das $\mathrm{PICS}^{14}$. Diante disso, este trabalho é um importante passo para se obter o aprimoramento e possibilitar a implementação oficial do ensino das PICS dentro do currículo 
médico. As PICS têm o potencial para ser importante ferramenta na ressignificação almejada para a educação médica ${ }^{5,8,13}$.

\section{CONCLUSÕES}

Os resultados obtidos por este estudo demonstraram que a comunidade acadêmica de Medicina tem pouco conhecimento sobre as PICS. Apesar disso, a maioria de docentes e discentes recomendaria o uso das PICS para seus pacientes e entende a importância delas para o SUS. A avaliação positiva do IMAQ evidencia que há interesse da comunidade acadêmica em aprofundar os conhecimentos relacionados às práticas integrativas, apesar de ser pouco expressiva, pela falta desse conteúdo no processo de formação.

É notória a carência teórico-prática dos discentes e docentes sobre a temática. A introdução das terapias complementares na base curricular do curso de Medicina repercutiria em um melhor debate de cunho educativo sobre a importância das PICS na graduação médica e contribuiria para uma melhor qualificação dos futuros profissionais em saúde para que estejam mais bem preparados para o cuidado integral, considerando o aumento da demanda por práticas integrativas e complementares para além da racionalidade biomédica.

\section{CONTRIBUIÇÃO DOS AUTORES}

Todos os autores contribuíram substancialmente para a concepção e o planejamento do estudo, a análise e interpretação dos dados, a elaboração do rascunho e a revisão crítica do conteúdo.

\section{CONFLITO DE INTERESSES}

Declaramos não haver conflito de interesses.

\section{FINANCIAMENTO}

Declaramos não haver financiamento.

\section{REFERÊNCIAS}

1. Brasil. Relatório do $1{ }^{\circ}$ Seminário Internacional de Práticas Integrativas e Complementares em Saúde - PNPIC. Série C. Projetos, programas e relatórios. Brasília: Ministério da Saúde; 2009 [access in 08 jun 2021]. Available from: http://bvsms.saude.gov.br/bvs/publicacoes/seminario_ praticas_integrativas_complementares_saude.pdf.

2. Badri M, Dissanayake DMRD, Kao FF. The promotion and development of traditional medicine. Geneva: World Health Organization; 1978 [access in 08 jun 2021]. Available from: http://whqlibdoc.who.int/trs/WHO_ TRS_622.pdf.

3. Brasil. Política Nacional de Práticas Integrativas e Complementares no SUS. 2a ed. Brasília: Ministério da Saúde; 2015 [access in 08 jun 2021]. Available from: http://bvsms.saude.gov.br/bvs/publicacoes/politica nacional_praticas_integrativas_complementares_2ed.pdf.
4. De Sousa IMC, Tesser CD. Medicina tradicional e complementar no Brasil: inserção no Sistema Único de Saúde e integração com a atenção primária. Cad Saude Publica. 2017;33(1):1-15 [access in 08 jun 2021]. Available from: http://cadernos.ensp.fiocruz.br/csp/artigo/13/medicina-tradicionale-complementar-no-brasil-insercao-no-sistema-unico-de-saude-eintegracao-com-a-atencao-primaria.

5. Aroucha EBL. Práticas Integrativas e Complementares: o interesse em formação dos profissionais da Estratégia de Saúde da Família [monografia]. Recife: Instituto Aggeu Magalhães, Fundação Oswaldo Cruz; 2012 [access in 08 jun 2021]. Available from: https://www.cpqam.fiocruz. br/bibpdf/2012aroucha-ebl.pdf.

6. Santos FAS, De Sousa IMC, Gurgel IGD, Bezerra AFB, De Barros NF. Política de práticas integrativas em Recife: análise da participação dos atores. Rev Saude Publica. 2011;45(6):1 154-9 [access in 08 jun 2021]. Available from: http://www. scielo.br/scielo.php?script=sci_arttext\&pid=S0034-89102011000600018.

7. Silverstein DD, Spiegel AD. Are physicians aware of the risks of alternative medicine? J Community Health. 2001;26(3):159-74 [access in 08 jun 2021]. Available from: https://pubmed.ncbi.nlm.nih.gov/11478563.

8. Dos Santos ACD. Elaboração e validação de uma matriz de competências em Práticas Integrativas e Complementares em Saúde para um programa de terceiro ano opcional de residência em Medicina de Família e Comunidade [dissertação]. Recife: Instituto Aggeu Magalhães, Fundação Oswaldo Cruz; 2019 [access in 08 jun 2021]. Available from: https://profsaude-abrasco.fiocruz.br/tcm/elaboracao-validacao-matrizcompetencias-praticas-integrativas-complementares-saude-programa.

9. Pinheiro LDSS. Validação de campo do Integrative Medicine Attitude Questionnaire (IMAQ) para uso no Brasil [dissertação]. Porto Alegre: Pontifícia Universidade Católica do Rio Grande do Sul; 2015 [access in 08 jun 2021]. Available from: http://tede2.pucrs.br/tede2/handle/tede/6325.

10. Greiner KA, Murray JL, Kallail KJ. Medical student interest in alternative medicine. J Altern Complement Med. 2000;6(3):231-4 [access in 08 jun 2021]. Available from: https://pubmed.ncbi.nlm.nih.gov/10890331.

11. Scheffer M, Cassenote A, Guilloux AGA, Miotto BA, Mainardi GM, Matijasevich $A$, et al. Demografia médica no Brasil 2018. São Paulo: Departamento de Medicina Preventiva da Faculdade de Medicina da USP, Conselho Regional de Medicina do Estado de São Paulo, Conselho Federal de Medicina; 2018 [access in 08 jun 2021]. Available from: https://jornal. usp.br/wp-content/uploads/DemografiaMedica2018.pdf.

12. Brasil.Portaria ${ }^{\circ} 702$, de 21 de março de 2018. Altera a Portaria deConsolidação no 2/GM/MS, de 28 de setembro de 2017, para incluir novas práticas na Política Nacional de Práticas Integrativas e Complementares - PNPIC. Diário Oficial da União; 56 mar 2018 [access in 08 jun 2021]. Available from: http:// bvsms.saude.gov.br/bvs/saudelegis/gm/2018/prt0702_22_03_2018.html.

13. Teixeira $M Z$, Lin CA. Educação médica em terapêuticas não convencionais. Rev Med (São Paulo). 2013;92(4):224-35 [access in 08 jun 2021]. Available from: https://www.revistas.usp.br/revistadc/article/view/80004.

14. Brasil. Resoluçãon ${ }^{\circ} 3$, de 20 de junho de 2014. Institui Diretrizes Curriculares Nacionais do Curso de Graduação em Medicina e dá outras providências. Diário Oficial da União; jun 2014 [access in 08 jun 2021]. Available from: http://portal.mec.gov.br/escola-de-gestores-da-educacao-basica/323secretarias-112877938/orgaos-vinculados-82187207/20138-ces-2014.

15. Giveon SM, Liberman N, Klang S, Kahan E. A survey of primary care physicians' perceptions of their patients' use of complementary medicine. Complement Ther Med. 2003;11(4):254-60 [access in 08 jun 2021]. Available from: https://pubmed.ncbi.nlm.nih.gov/15022662.

16. Christensen MC, De Barros NF. Medicinas alternativas e complementares no ensino médico: revisão sistemática. Rev Bras Educ Med. 2010;34(1):97105 [access in 08 jun 2021]. Available from: https://www.scielo.br/scielo. php?pid=S0100-55022010000100012\&script=sci_abstract\&tlng=pt.

17. Freitas $H$, Oliveira $M$, Saccol $A Z$, Moscarola J. O método de pesquisa survey. Revista de Administração da USP. 2000;3(3):105-12 [access in 08 jun 2021]. Available from: http://www.ufrgs.br/gianti/files/artigos/2000/2000_092 RAUSP.PDF.

18. Cendón BV, Ribeiro NA, Chaves CJ. Pesquisas de survey: análise das reações dos respondentes. Informação \& Sociedade: Estudos. 2014;24(3):29-48 [access in 08 jun 2021]. Available from: https://periodicos.ufpb.br/ojs/ index.php/ies/article/view/19963.

This is an Open Access article distributed under the terms of the Creative Commons Attribution License, which permits unrestricted use, distribution, and reproduction in any medium, provided the original work is properly cited. 